\title{
Odotopia and intraoperative brain mapping: implications for management of patients with diffuse low-grade gliomas
}

\begin{abstract}
Recent methods for diagnosis and surgery have really changed the relationships between structure and functioning in the brain, so bringing to a holistic vision and explicating the concept of odotopia with regard to gray and white matter interactions. Accordingly, the concept of brain plasticity and the surgical treatment have been revised in the light of new views as well, bringing to a new insight for multimodal management of diffuse low-grade gliomas.
\end{abstract}

Keywords: low-grade gliomas, odotopia, connectome, brain mapping, direct intraoperative stimulation
Volume 7 Issue I - 2017

\section{Fabio Barone}

Department of Neurosurgery, Cannizzaro General Hospital, Italy

Correspondence: Fabio Barone, Department of Neurosurgery, Cannizzaro General Hospital,Via Messina 829, 95126 Catania, Italy,Email Fabio.barone@aoec.it;fbarone2009@alice.it

Received: April 15, 2017 | Published: June 23, 2017

\section{Introduction}

The neuro-oncological patients implies very often frustration for specialists who deal with them.

These cases comprise glial tumours and, either low or high grade histology, invariably progress sooner or later to death despite maximal optimization of the treatment.

From a surgical point of view, last years have showed more and more satisfying results thanks to methods defining tumour's exact location and identifying the function of the brain in the tumoral area. The aim is to optimize the removal and minimizing permanent postoperative deficits to bring the patient to the adjuvant treatment as well as possible, to guarantee of a good compliance. In fact, considering that it is still impossible to heal the patient, each treatment must aim to get the best possible result (the most durable disease control) keeping the residual quality of life at the top.

The surgical technique has gone on this way during last years, developing methods to be able to "map" functions of the human brain which is nowadays considered not only as a set of highly specific cortical areas but as holistic entity, a set of gray and white matter which are strictly connected each other.

\section{Discussion}

Studying the relationships between the components of the central nervous system have contributed a lot to defining the concept of all the "brain", determining a progressive change of view with regard to explain its working concept. From "localizationism", where every function had been tightly linked to a specific cortical area, we have passed to "connectionism", where instead the function is dependent on the existing network between different cerebral areas through white matter fascicles, according to neural networks model: the travelling informations (biological or artificial) are delivered for all the web nodes and not in a single "place"; by this way it cannot target to a precise point of the system and, at the same time, it cannot say that this point has a specific information or it performs a specific task. Such a vision bring us back to the already described model by Rumelhart, at the end of " $80 \mathrm{yrs}$, as "Parallel Distributed Processing (PDP)".

This view payed more attention to the subcortical white matter which must be kept as intact as possible during surgery to preserve the function. Indeed scientific reports regarding stroke have described lesions resulting by subcortical white matter injury as worse than cortical injury.

From the beginning of ' $90 \mathrm{yrs}$ the connectionistic view took advantage of the progressing radiological methods (DTI fiber trackingMR) and intraoperative surgical methods of functional "mapping",finding practical reflection in clinical practice and making a revision of the disorders involving cognitive functions, even from a prognostic point of view. All the above described brought to a logical evolution of the "connectionism", giving a similar role for a functional deficit, which has been caused, for instance, by an infiltrating tumour, to the cortex and, at the same time, to the corresponding axonal connection: the term "odotopia" (from Greek odòs, road, and tòpos, place) is so introduced, in order to indicate a set of functional processes comprising in turn on the one hand a "topological" mechanism, expliciting cortical disfunctions which have been derived by a deficit, hyperactivation or both and, the other part, a "odological" mechanism referring to disfunctions which have been caused by white matter fascicles lesions, for disconnection, hyperconnection or both.

So this mechanism may explain deficits which have been caused by cortical or subcortical lesions, involving more or less the white matter. In other words it is mandatory considering the large-scale cortico-subcortical network to understand physiology and the functional result of a lesion, with deficits depending on the location and extension of the injury (just cortical, solo subcortical or both).

Odotopic vision has been recently more and more considered because of specific neuroanatomical studies aimed to "materialize" superior cognitive functions. In particular, regarding the language, the old localizationistic views have been upset in place of a language as result of a "PDP" by a defined and diffused set of network of neurons, instead of individual areas. Going over the "serial model" of the language, where it is imperative to complete a single process before the information may reach another level of "processing", new "independent" network models make possible different processes may be simultaneously executed, becoming connected each other by interactive "feedbacks".

DTIMR images and intraoperative cortical and subcortical direct electric stimulation (DES) concretely interact with such a theoretic vision, supporting the large-scale linguistic neural network models 
which had been already thought by Mesulam at the early ' $90 \mathrm{yrs}$. In particular, for language it is now acquired that at least two parallel pathways are active, a dorsal phonological stream and a semantic ventral stream, both of them formed by associative fascicles of subcortical white matter and converging to a final common pathway bringing to the real language (Figure 1,2). All this network is also modulated by the corticostriotalamocortical and corticopontocerebellotalamocortical loops to regulate the rhythms. ${ }^{3,4}$

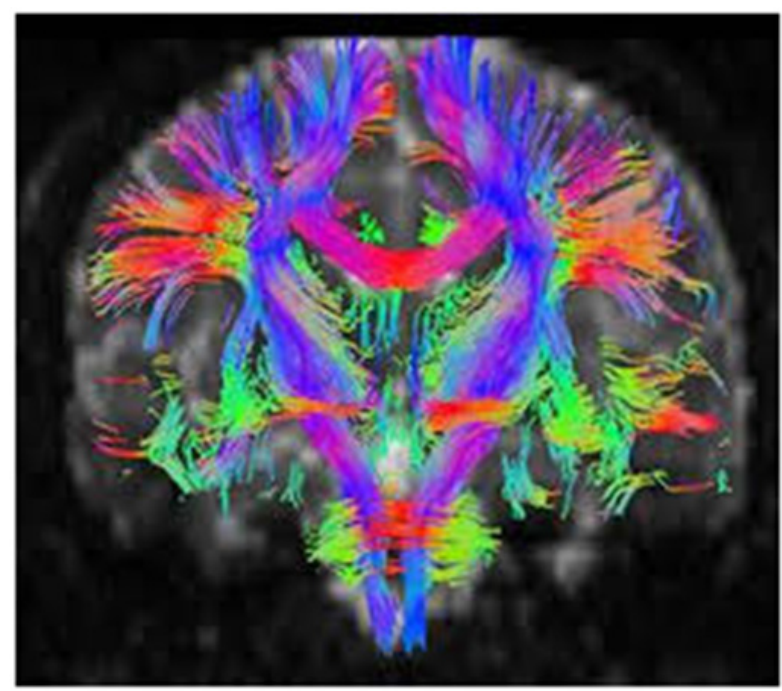

Figure I DTI imaging of associative and projective tracts.

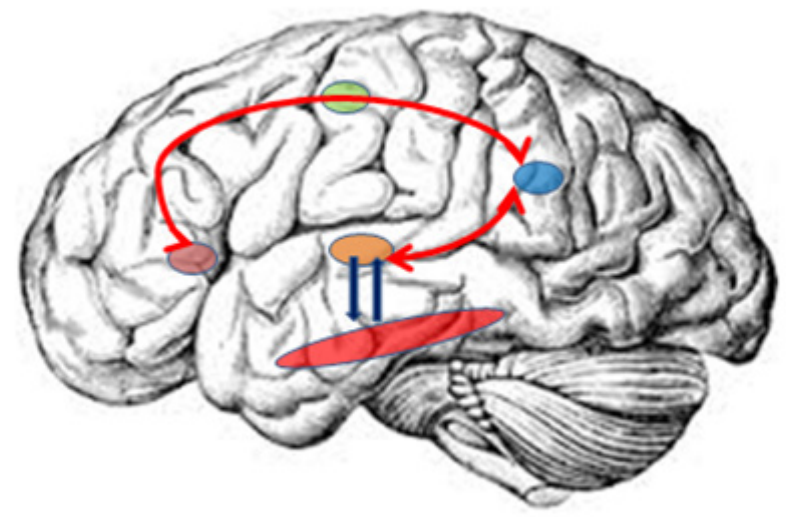

Figure 2 Schematic drawing of the dual stream of language model; the ventral stream (blue arrows) flows through the anterior and middle temporal lobe (red ellipsoid).The dorsal stream (red curved line) connects ventral premotor area with parietotemporal area through motor area.

In fact intraoperative DES of cerebral region (cortical or subcortical), eventually performed in an awake patient, will never be corresponding to a stimulation of a specific site but of a real "gate" of a complex network; because of that it allows studying a large neurofunctional circuit which is very relevant from a clinical point of view, being prognostically important in order to predict eventual transient or permanent loss of a function after surgery. From that, we can understand why intraoperative DES allowed a substantial increase in the survival and quality of life of patients: maximizing resection until the functional boundaries are reached. ${ }^{5,6}$

By this assessment it is possible to explain, why some areas, considered as functionally essential, are involved by a tumour (or surgically removed) without showing any deficit: in these cases the functional compensation of the above mentioned networks plays a relevant role, de facto making real the concept of neuroplasticity and implying a specific patient-tailored decisional pattern for management of the disease. Neuroplasticity can be defined as a "continuous process allowing short-term, middle-term and long-tern remodelling of neurosynaptic maps, to optimize the functioning of brain networks".?

Accepting the large number of the last ones brings to establish the concept of connectome, a complex of parallel subcircuits able to compensate themselves after brain injury. ${ }^{8}$ This framework contributed so much to refine the contemporary concepts of language organization as we above described. So in order to prevent permanent deficits that may decrease quality of life, a patient with a low-grade glioma, with a constant but slow growth until to change to anaplasia, may take advantage of serial incomplete surgical removals which, if on the one hand may be considered therapeutically disappointing, the other part may open the way to a "multistaged" surgical approach, supported by serial functional neuroimaging to show the changes occurring after surgery and depending on the plasticity. ${ }^{9-1}$

By this way it is possible to remove in another future procedure a portion of infiltrated tissue, functionally active at that time and that, because of the plasticity, may become inactive with time. This allows the patient to get a more durable control of the disease, keeping a good level of quality of life and not being compelled to adjuvant treatment and its eventual toxicity.

\section{Conclusion}

So, the central nervous system is not so different than other systems or tissues of the human body, because the neuroplasticity may be considered a proper repairing mechanism; the Specialists who are called to follow its evolving process should be so wise and competent to drive and profit by it in order to get the best benefit for the patients.

\section{Acknowledgments}

None.

\section{Conflicts of interest}

None.

\section{Funding}

None.

\section{References}

1. Mandonnet E, Winkler PA, Duffau H. Direct electrical stimulation as an input gate into brain functional networks: principles, advantages and limitations. Acta Neurochir (Wien). 2010; 152(2):185193.

2. Chang E, Raygor K, Berger M. Contemporary model of language organization. An overview for neurosurgeons. $J$ Neurosurg. 2015;122(2):250261.

3. Rumelhart DE, McClelland JL and the PDP Research Group. Parallel Distributed Processing: Explorations in the Microstructure of Cognition. Volume 1: Foundations. MIT Press, Cambridge, MA, USA. 1986.

4. Catani M. From hodology to function. Brain. 2007;130(3):602605.

5. Duffau H. Brain hodotopy: New insights provided by intrasurgical mapping. In: Duffau H (Ed.), Brain Mapping From Neural Basis of Cognition to Surgical Applications. Elsevier WienNew York, USA. 2011. p.335347.

6. De Witt Hamer PC, Robles SG, Zwinderman AH, et al. Impact of intraoperative stimulation brain mapping on glioma surgery outcome: a meta-analysis. J Clin Oncol. 2012;30(20):2559-2565.

7. Duffau H. A new philosophy in surgery for diffuse low-grade glioma (DLGG): oncological and functional outcome. Neurochirurgie. 2013;59(1):2-8. 
8. Smith SM, Vidaurre D, Beckmann CF, et al. Functional connectomics from resting-state fMRI. Trends Cogn Sci. 2013;17(12):666-682.

9. Duffau H, Taillandier L. New concepts in the management of diffuse low-grade: proposal of a multistage and individualized therapeutic approach. Neuro Oncol. 2015;17(3):332-342.
10. Martino J, Taillandier L, Moritz-Gasser S, et al. Re-operation is a safe and effective therapeutic strategy in recurrent WHO grade II gliomas within eloquent areas. Acta Neurochir. 2009;151(5):427-436.

11. Ghinda CD, Duffau H. Network plasticity and intraoperative mapping for personalized multimodal management of diffuse low-grade gliomas. Front Surg. 2017;4:3. 